\title{
Low-power laser irradiation promotes the proliferation and osteogenic differentiation of human periodontal ligament cells via cyclic adenosine monophosphate
}

\author{
Jyun-Yi Wu ${ }^{1}$, Chia-Hsin Chen ${ }^{2,3,4}$, Li-Yin Yeh ${ }^{5}$, Ming-Long Yeh ${ }^{1}$, Chun-Chan Ting ${ }^{6}$ and Yan-Hsiung Wang ${ }^{6,7}$
}

Retaining or improving periodontal ligament (PDL) function is crucial for restoring periodontal defects. The aim of this study was to evaluate the physiological effects of low-power laser irradiation (LPLI) on the proliferation and osteogenic differentiation of human PDL (hPDL) cells. Cultured hPDL cells were irradiated $(660 \mathrm{~nm})$ daily with doses of $0,1,2$ or $4 \mathrm{~J} \cdot \mathrm{cm}^{-2}$. Cell proliferation was evaluated by the 3-[4,5-dimethylthiazol-2-yl]-2,5-diphenyltetrazolium bromide (MTT) assay, and the effect of LPLI on osteogenic differentiation was assessed by Alizarin Red S staining and alkaline phosphatase (ALP) activity. Additionally, osteogenic marker gene expression was confirmed by real-time reverse transcription-polymerase chain reaction (RT-PCR). Our data showed that LPLI at a dose of $2 \mathrm{~J} \cdot \mathrm{cm}^{-2}$ significantly promoted hPDL cell proliferation at days 3 and 5 . In addition, LPLI at energy doses of 2 and $4 \mathrm{~J} \cdot \mathrm{cm}^{-2}$ showed potential osteogenic capacity, as it stimulated ALP activity, calcium deposition, and osteogenic gene expression. We also showed that cyclic adenosine monophosphate (cAMP) is a critical regulator of the LPLI-mediated effects on hPDL cells. This study shows that LPLI can promote the proliferation and osteogenic differentiation of hPDL cells. These results suggest the potential use of LPLI in clinical applications for periodontal tissue regeneration.

International Journal of Oral Science (2013) 5, 85-91; doi:10.1038/ijos.2013.38; published online 21 June 2013

Keywords: cell proliferation; cyclic adenosine monophosphate; human periodontal ligament cells; low-power laser irradiation; osteogenic differentiation

\section{INTRODUCTION}

The periodontium consists of four defined tissues, including the gingiva, cementum, alveolar bone and periodontal ligament, which are supporting tissues that surround the teeth. Among these structures, the periodontal ligament $(\mathrm{PDL})$ is the connective tissue located in the tooth root that connects the tooth to the alveolar bone and gingiva. With respect to its physiological function, the PDL not only provides physical resistance to the impact of occlusal forces but also regulates the remodeling of the alveolar bone by transmitting forces. ${ }^{1}$ Furthermore, the PDL plays a critical role during orthodontic tooth movement. An exertion of sufficient orthodontic force causes tooth migration by tightly regulating the alveolar bone remodeling process. Under these conditions, alveolar bone resorption occurs on the pressured side, and new bone formation occurs on the tensile side. ${ }^{2}$

Periodontal diseases, such as gingivitis and periodontitis, are common chronic infectious diseases. Periodontal disease can cause swelling and pain and may induce irreversible destruction of periodontal tissue and perhaps even tooth loss. ${ }^{3}$ Clinically, ultrasonic scaling and root planing are conventional periodontal therapeutic measures used to eliminate calculus and bacteria-infected plaques. ${ }^{4}$ However, these treatments only remove causative agents and have few benefits for the regeneration of a degraded periodontium.

Successful regeneration of the periodontium requires the reestablishment of previously lost tissues. In recent years, many treatment modalities have been developed to improve periodontium regeneration, including osseous grafting and guided tissue regeneration. ${ }^{5-6}$ In addition, low-power laser irradiation (LPLI) has been used for nonthermal and non-invasive physical treatment. LPLI has been used in medical applications to provide pain relief, ${ }^{7-8}$ reduce inflammatory mediators ${ }^{9-10}$ and accelerate the wound healing process. ${ }^{1-12}$ Until now, the effects of LPLI on PDL cells have not been well known, though some studies have shown that LPLI can promote the proliferation and differentiation of several cell types in vitro. ${ }^{13-15}$ Considering the biologically beneficial effects induced by LPLI, the use of LPLI instrumentation during the periodontium regeneration process may be therapeutically useful. However, the mechanism by which LPLI induces a cellular response and the optimal conditions for LPLI therapy, such as the fluency and wavelength, are unclear.

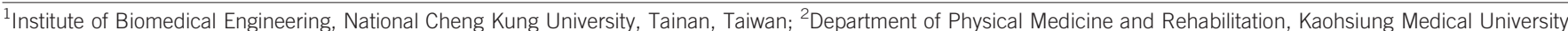

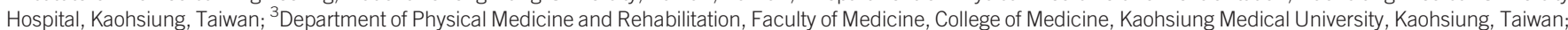

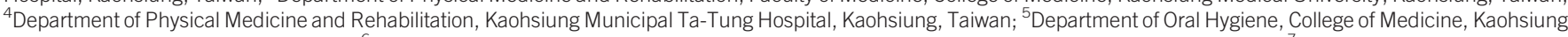

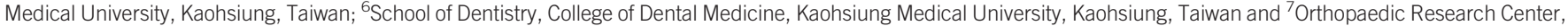
College of Medicine, Kaohsiung Medical University, Kaohsiung, Taiwan

Correspondence: Dr YH Wang, School of Dentistry, College of Dental Medicine, Kaohsiung Medical University, 100, Shih-Chuan 1st Road, Kaohsiung 80708, Taiwan

E-mail: yhwang@kmu.edu.tw

Received 6 November 2012; accepted 16 May 2013 
The aim of this study was to determine the effects and potential mechanism of LPLI on the proliferation and osteogenic differentiation of PDL cells with the expectation that LPLI could be clinically administered for the regeneration of periodontal tissue in the future.

\section{MATERIALS AND METHODS}

\section{Cell culture}

Human PDL (hPDL) cells were purchased from Lonza Group, Ltd (Basel, Switzerland). The hPDL cells were cultured and expanded in medium consisting of keratinocyte serum-free medium (Gibco-BRL, Rockville, MD, USA) and Dulbecco's modified Eagle's medium with low glucose (Gibco-BRL, Rockville, MD, USA) in a 1:1 ratio, which we named BK medium. The medium contained bovine pituitary extract $(25 \mathrm{mg})$, human recombinant epidermal growth factor protein $(2.5 \mu \mathrm{g}), 0.2 \mathrm{mmol} \cdot \mathrm{L}^{-1} \mathrm{~N}$-acetyl-cysteine (Sigma-Aldrich, St Louis, MO, USA), $0.2 \mathrm{mmol} \cdot \mathrm{L}^{-1} \mathrm{~L}$-ascorbic acid 2-phosphate (SigmaAldrich, St Louis, MO, USA), 5\% fetal bovine serum (SAFC Biosciences, St Louis, MO, USA), $1 \%$ penicillin/streptomycin (Gibco-BRL, Rockville, MD, USA) and $0.06 \mu \mathrm{g} \cdot \mathrm{mL}^{-1}$ insulin (Sigma-Aldrich, St Louis, MO, USA). ${ }^{16}$ The cells were cultured in a humidified incubator with $5 \% \mathrm{CO}_{2}$ at $37^{\circ} \mathrm{C}$. The medium was changed every other day.

\section{Osteogenic induction of hPDL cells}

hPDL cells were cultured for 2 days to achieve $80 \%$ confluence, and the media was then replaced with osteo-induction medium (OIM). OIM consists of Dulbecco's modified Eagle's medium supplemented with $10^{-7} \mathrm{~mol} \cdot \mathrm{L}^{-1}$ dexamethasone (Sigma-Aldrich, St Louis, MO, USA), $50 \mu \mathrm{mol} \cdot \mathrm{L}^{-1} \mathrm{~L}$-ascorbic acid 2-phosphate (Sigma-Aldrich, St Louis, MO, USA) and $10 \mathrm{mmol} \cdot \mathrm{L}^{-1} \beta$-glycerophosphate (SigmaAldrich, St Louis, MO, USA), as described by Wang et al. ${ }^{17}$ The culture medium was changed every 2 days.

\section{Low-power laser irradiation}

A gallium-aluminum-arsenide (GaAlAs) 660-nm red laser (Transverse Ind. Co., Ltd, Taipei, Taiwan) was used in this study. The maximum power output was $70 \mathrm{~mW}$, and the power was adjusted to achieve the intended energy exposure for the target. The distance between the laser source and the cells was $3 \mathrm{~cm}$. Under these conditions, the power density was $15.17 \mathrm{~mW} \cdot \mathrm{cm}^{-2}$, and the cells were exposed to laser irradiation for 66, 132 and $264 \mathrm{~s}$ to apply energy densities of 1,2 and $4 \mathrm{~J} \cdot \mathrm{cm}^{-2}$, respectively. The cells were irradiated daily at room temperature on a clean bench. The non-irradiated control cells were incubated at room temperature for the same duration.

\section{SQ22536 preparation}

SQ22536 (Sigma-Aldrich, St Louis, MO, USA) is an adenylyl cyclase inhibitor that inhibits cyclic adenosine monophosphate (cAMP) production. SQ22536 was dissolved in dimethylsulfoxide (J. T. Baker, Philipsburg, NJ, USA) and added to BK medium at a final concentration of $100 \mu \mathrm{mol} \cdot \mathrm{L}^{-1}$.

\section{3-[4,5-dimethylthiazol-2-yl]-2,5-diphenyltetrazolium bromide assay}

To estimate the proliferation of hPDL cells, $4 \times 10^{3}$ cells were seeded into 96-well culture plates for 3-[4,5-dimethylthiazol-2-yl]-2,5-diphenyltetrazolium bromide (MTT) (Sigma-Aldrich, St Louis, MO, USA) assays. Briefly, $40 \mu \mathrm{L}$ of MTT reagent was added to each well, and the plate was then incubated at $37{ }^{\circ} \mathrm{C}$ for $4 \mathrm{~h}$. Subsequently, $40 \mu \mathrm{L}$ of dimethylsulfoxide was added to each well to dissolve the formazan produced from MTT. After 10 -min incubation at $37{ }^{\circ} \mathrm{C}$ to allow for a complete reaction, the plate was measured using an enzyme-linked immunosorbent assay (ELISA) plate reader (model 680; Bio-Rad, Hercules, CA, USA) at $595 \mathrm{~nm}$.

\section{Lactate dehydrogenase leakage}

Lactate dehydrogenase (LDH) leakage was measured to quantify cytotoxicity using the Cytotoxicity Detection kit (Roche, Mannhein, Switzerland). The supernatants and cells in culture were assayed based on the manufacturer's guidelines. Absorbance was measured using an ELISA reader at $490 \mathrm{~nm}$. The $\mathrm{LDH}$ leakage was calculated by determining the ratio of the absorbance of the supernatant to that of the supernatant and cell lysate combined.

\section{Alkaline phosphatase activity}

For the alkaline phosphatase (ALP) activity assay, hPDL cells were seeded at $5 \times 10^{4}$ cells per well in 12-well culture plates and harvested after 3 and 5 days of culture in OIM. The collected cells were washed twice with phosphate-buffered saline (Bio-Tech, Santa Cruz, CA, USA) and lysed in $0.2 \%$ Triton X-100 lysis buffer. The cell lysates were then assayed for ALP activity following the manufacturer's instructions (Tropix; Applied Biosystems, Bedford, MA, USA) and measured using a TopCount scintillation counter in chemiluminescent mode (Packard Biosciences, Waltham, MA, USA). The protein concentration was determined using a BCA Protein Assay Kit (Novagen, Darmstadt, Germany) according to the manufacturer's instructions. The ALP activity was normalized to the protein concentration for each sample.

\section{Alizarin Red S staining}

We estimated the amount of calcium that was deposited during matrix mineralization with Alizarin Red S staining. hPDL cells were seeded at $5 \times 10^{4}$ cells per well in 12 -well culture plates and harvested after 7, 10 and 14 days of culture in OIM. The cells were washed twice with phosphate-buffered saline and fixed in 10\% formaldehyde (Tonyar Biotech Inc., Taoyuan, Taiwan) for $10 \mathrm{~min}$. The cells were then washed twice with deionized water and stained with Alizarin Red S (SigmaAldrich, St Louis, Mo, USA) for $10 \mathrm{~min}$ at room temperature. After staining the cells, excess dye was removed by gently rinsing with running water. Dye from cells that were stained red, which is typically indicative of calcium deposits, was extracted for quantification using $200 \mu \mathrm{L}$ of $10 \%$ glacial acetic acid at $60{ }^{\circ} \mathrm{C}$ for $1 \mathrm{~h}$. The amount of extracted Alizarin Red was determined using an ELISA plate reader at $490 \mathrm{~nm}$.

\section{Real-time polymerase chain reaction}

For real-time polymerase chain reaction (PCR) analysis, hPDL cells were seeded at $5 \times 10^{4}$ cells per well in 12-well culture plates and harvested after 3 or 5 days of culture in OIM. The cells were washed twice with phosphate-buffered saline. Total RNA was extracted using the TRIzol reagent (Gibco-BRL, Rockville, MD, USA) according to the manufacturer's instructions. The RNA pellet was washed once with $75 \%$ ethanol and once with $100 \%$ ethanol for dehydration. The RNA was dissolved in diethylpyrocarbonate-treated water, and spectrophotometry was used to quantify the RNA concentration by measuring the absorbance (ND-1000; NanoDrop Technologies Inc., Wilmington, DE, USA) at 260 and $280 \mathrm{~nm}$. First-strand cDNA was reverse transcribed by adding $1 \mu \mathrm{g}$ of RNA to a mixture of Moloney murine leukemia virus reverse transcriptase and oligo-dT primers, and quantitative real-time PCR was performed using a Bio-Rad iQ5 real-time detection system (Bio-Rad, Hercules, CA, USA). PCR reactions were 
performed in a $12.5 \mu \mathrm{L}$ solution containing cDNA, forward and reverse primers, and SYBR Green Real-time PCR Master Mix (Toyobo, Osaka, Japan). Primer pairs for the following human genes were used: glyceraldehyde 3-phosphate dehydrogenase (GAPDH; 5'-GAA GGT GAA GGT CGG AGT CA-3' forward and 5'-GAA GAT GGT GAT GGG ATT TC-3' reverse), bone morphogenetic protein 2 (BMP2; 5'-CGA ATG ACT GGA TTG TGG CT-3' forward and 5'-TGA GTT CTG TCG GGA CAC AG-3' reverse), runt-related transcription factor 2 (RUNX2; 5' -AGA TGG GAC TGT GGT TAC TG-3' forward and 5' GTA GCT ACT TGG GGA GGA TT-3' reverse), osteocalcin (OC; $5^{\prime}$ GTG CAG AGT CCA GCA AAG GT-3' forward and $5^{\prime}$-CGA TAG GCC TCC TGA AAG C-3' reverse) and ALP (5'-AAC TTC CAG ACC ATT GGC TTG A-3' forward and 5' -TTG CCG CGT GTC GTG TT-3' reverse). Relative mRNA expression levels were calculated based on the threshold cycle $\left(C_{\mathrm{t}}\right)$ value of each PCR product and normalized to a housekeeping gene $(G A P D H)$ using the comparative $C_{\mathrm{t}}$ method.

\section{Statistical analysis}

SPSS version 17.0 was used for the statistical analyses. The results are expressed as the mean \pm standard deviation. Statistically significant differences were determined using an analysis of variance followed by the post hoc Tukey's test for multiple comparisons. A $P$-value less than 0.05 was considered statistically significant.

\section{RESULTS}

\section{LPLI promotes the proliferation of hPDL cells}

The proliferation of hPDL cells after exposure to LPLI was measured using an MTT assay. After irradiation with 1 and $2 \mathrm{~J} \cdot \mathrm{cm}^{-2}$, the MTT activity was significantly increased in LPLI-treated cells compared with the control cells at days 3 and 5 (Figure 1a). Moreover, no significantly different changes were found between the control group and the group treated with to the highest LPLI dose $\left(4 \mathrm{~J} \cdot \mathrm{cm}^{-2}\right.$ ) (Figure 1a) (day 1: control, $0.087273 ; 1 \mathrm{~J} \cdot \mathrm{cm}^{-2}, 0.087417 ; 2 \mathrm{~J} \cdot \mathrm{cm}^{-2}, 0.088385$; $4 \mathrm{~J} \cdot \mathrm{cm}^{-2}, 0.084$ 167; day 3: control, 0.205 435; $1 \mathrm{~J} \cdot \mathrm{cm}^{-2}, 0.214692$; $2 \mathrm{~J} \cdot \mathrm{cm}^{-2}, 0.215217 ; 4 \mathrm{~J} \cdot \mathrm{cm}^{-2}, 0.212714$; day 5: control, 0.344250 ; $\left.1 \mathrm{~J} \cdot \mathrm{cm}^{-2}, 0.375083 ; 2 \mathrm{~J} \cdot \mathrm{cm}^{-2}, 0.378080 ; 4 \mathrm{~J} \cdot \mathrm{cm}^{-2}, 0.336250\right)$.

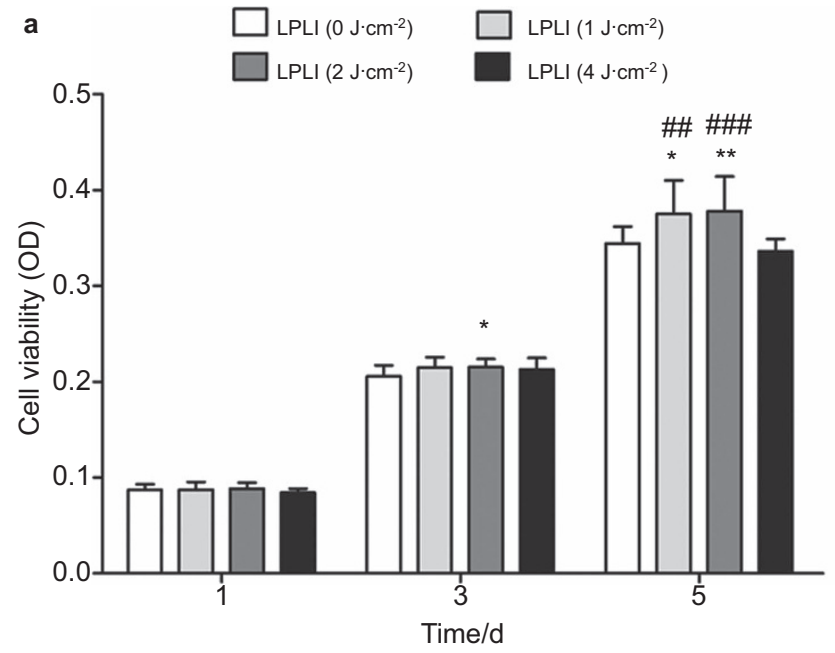

In addition, the cytotoxic effects of LPLI on hPDL cells were measured by analyzing LDH leakage. The results of this analysis showed that LPLI treatment did not induce cytotoxic effects in hPDL cells at any of the irradiation energy doses (Figure 1b). These results indicate that LPLI does not induce cytotoxic effects and that energy doses less than $4 \mathrm{~J} \cdot \mathrm{cm}^{-2}$ are more suitable for hPDL cell growth.

\section{LPLI enhances hPDL cell osteogenic differentiation}

We used ALP activity and Alizarin Red S staining to evaluate the effects of LPLI on the osteogenic differentiation of hPDL cells. hPDL cells cultured in OIM showed a higher ALP activity when compared with cells cultured in BK media (negative control). Interestingly, the ALP activity of hPDL cells cultured in OIM was significantly enhanced by an LPLI dose of $2 \mathrm{~J} \cdot \mathrm{cm}^{-2}$ on days 3 and 5 , although no additional increase was observed at $4 \mathrm{~J} \cdot \mathrm{cm}^{-2}$ (Figure $2 \mathrm{a}$ ).

Furthermore, LPLI promoted osteogenic differentiation in a dosedependent manner after culturing the cells for 7, 10 and 14 days (Figure 2b). The quantification of these cells by Alizarin Red S staining is shown in Figure 2c. hPDL cells cultured in OIM displayed mineral deposits compared with the BK-negative control group at these three time points. After LPLI at 2 and $4 \mathrm{~J} \cdot \mathrm{cm}^{-2}$, the staining intensity increased 1.16- and 1.17-fold, respectively, on day 7; 1.18- and 1.27fold, respectively, on day 10; and 1.12- and 1.13-fold, respectively, on day 14 , relative to non-irradiated hPDL cells cultured in OIM.

\section{LPLI induces the expression of osteogenic genes}

Next, we determined the gene expression levels of BMP2, OC, RUNX2 and $A L P$ by real-time RT-PCR. BMP2 expression significantly increased following the treatment of hPDL cells with LPLI at 2 and $4 \mathrm{~J} \cdot \mathrm{cm}^{-2}$ on day 3 . However, this increase was observed only for the $2 \mathrm{~J} \cdot \mathrm{cm}^{-2}$ dose on day 5 (Figure $3 \mathrm{a}$ ). A, similar pattern was also observed for $O C$ (Figure 3b) and RUNX2 (Figure 3c), in that LPLI at both energy doses $\left(2\right.$ and $\left.4 \mathrm{~J} \cdot \mathrm{cm}^{-2}\right)$ significantly increased the gene expression on day 3 , but no difference between the groups was found on day 5. Finally, LPLI increased ALP expression only at the energy dose of $2 \mathrm{~J} \cdot \mathrm{cm}^{-2}$ on days 3 and 5 ; however, the difference in ALP gene expression at the $4 \mathrm{~J} \cdot \mathrm{cm}^{-2}$ dose was not statistically significant

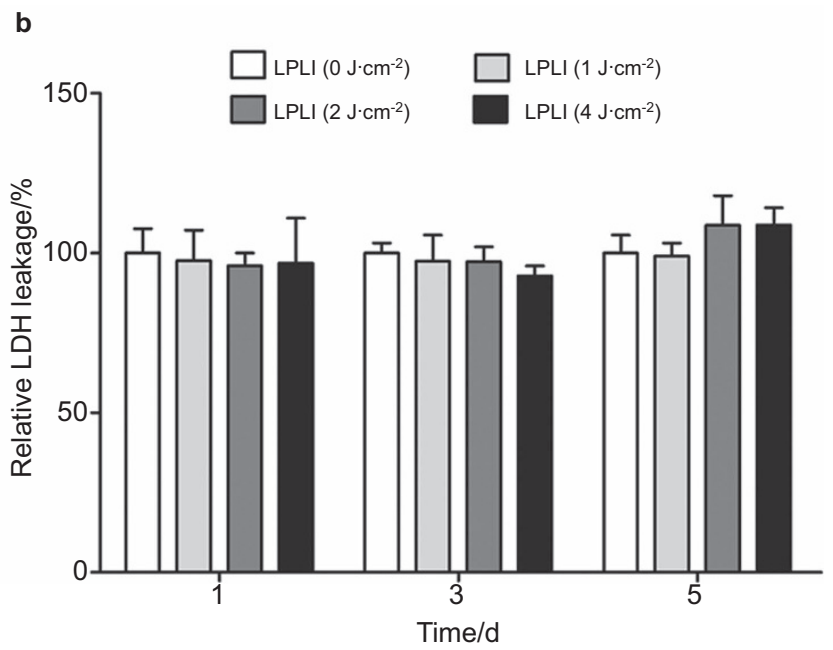

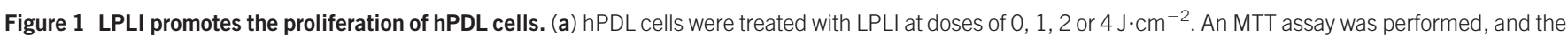

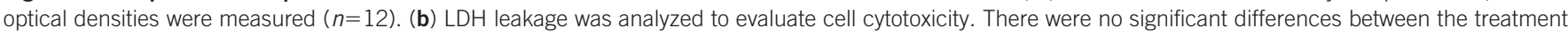

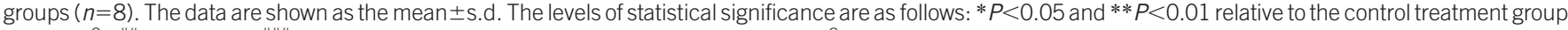

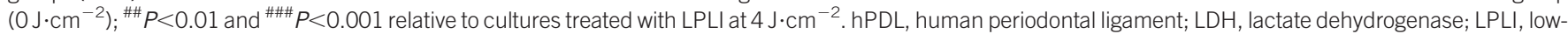
power laser irradiation; MTT, 3-[4,5-dimethylthiazol-2-yl]-2,5-diphenyltetrazolium bromide; OD, optical density. 

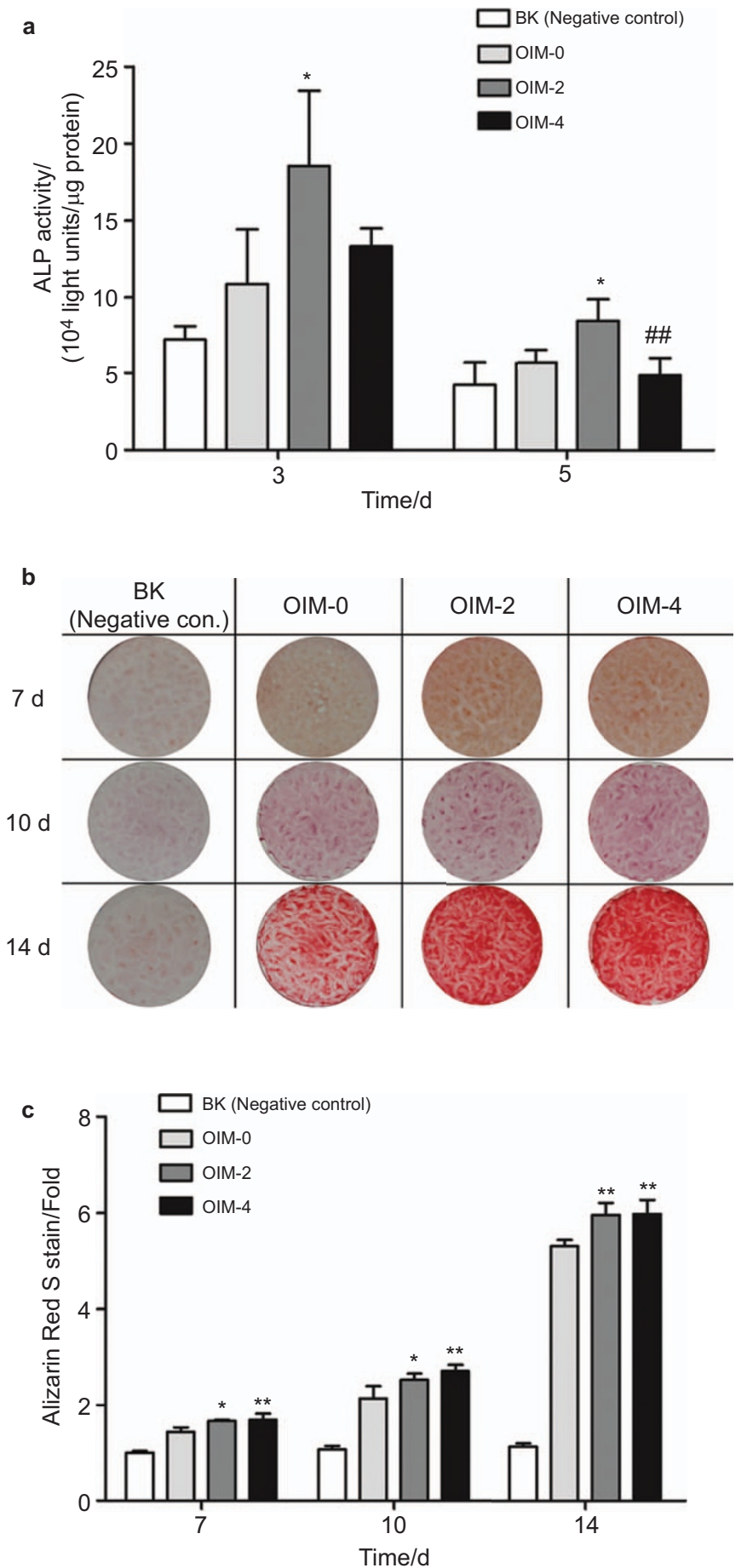

Figure 2 LPLI increased the osteogenic differentiation of hPDL cells. hPDL cells were divided into the following four groups: negative control, which was cultured in BK medium; OIM-0, which was cultured in OIM only; OIM-2, which was cultured in OIM and treated with LPLI at $2 \mathrm{~J} \cdot \mathrm{cm}^{-2}$; and OIM-4, which was cultured in OIM and treated with LPLI at $4 \mathrm{~J} \cdot \mathrm{cm}^{-2}$. (a) The ALP activity of the hPDL cells was analyzed on days 3 and 5 , as described in the section on 'Materials and methods' $(n=6)$. (b) hPDL cells were cultured for 7, 10 and 14 days. After fixation, the cells were stained with Alizarin Red S ( $n=6$ ). (c) Relative quantification of Alizarin Red S based on the staining shown in $\mathbf{a}$. The data are shown as the mean \pm s.d., and the levels of statistical significance are indicated as follows: $* P<0.05$ and $* * P<0.01$ relative to the OIM-0 group; ${ }^{\#} P<0.05$ relative to the OIM-2 group. ALP, alkaline phosphatase; hPDL, human periodontal ligament; LDH, lactate dehydrogenase; LPLI, lowpower laser irradiation; OIM, osteo-induction medium.
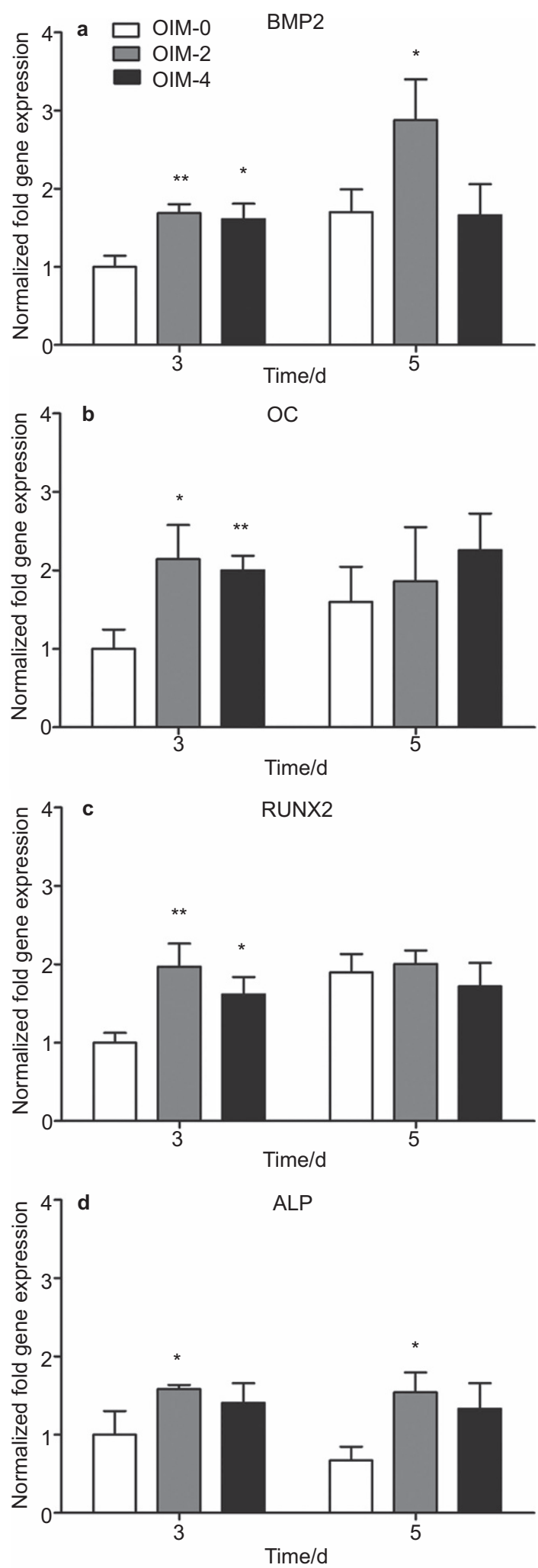

Figure $3 \mathrm{LPLI}$ enhanced the expression of osteogenic marker genes. The mRNA expression of BMP2 (a), OC (b), RUNX2 (c) and $A L P(\mathbf{d})$ was analyzed by the $2^{-\Delta C_{\mathrm{T}}}$ method and normalized to the OIM-0 group. The data are shown as the mean \pm s.d. $(n=4)$, and the levels of statistical significance are indicated as follows: $* P<0.05$ and $* * P<0.01$ relative to the OIM-0 group. ALP, alkaline phosphatase; LPLI, low-power laser irradiation; OIM, osteo-induction medium. 

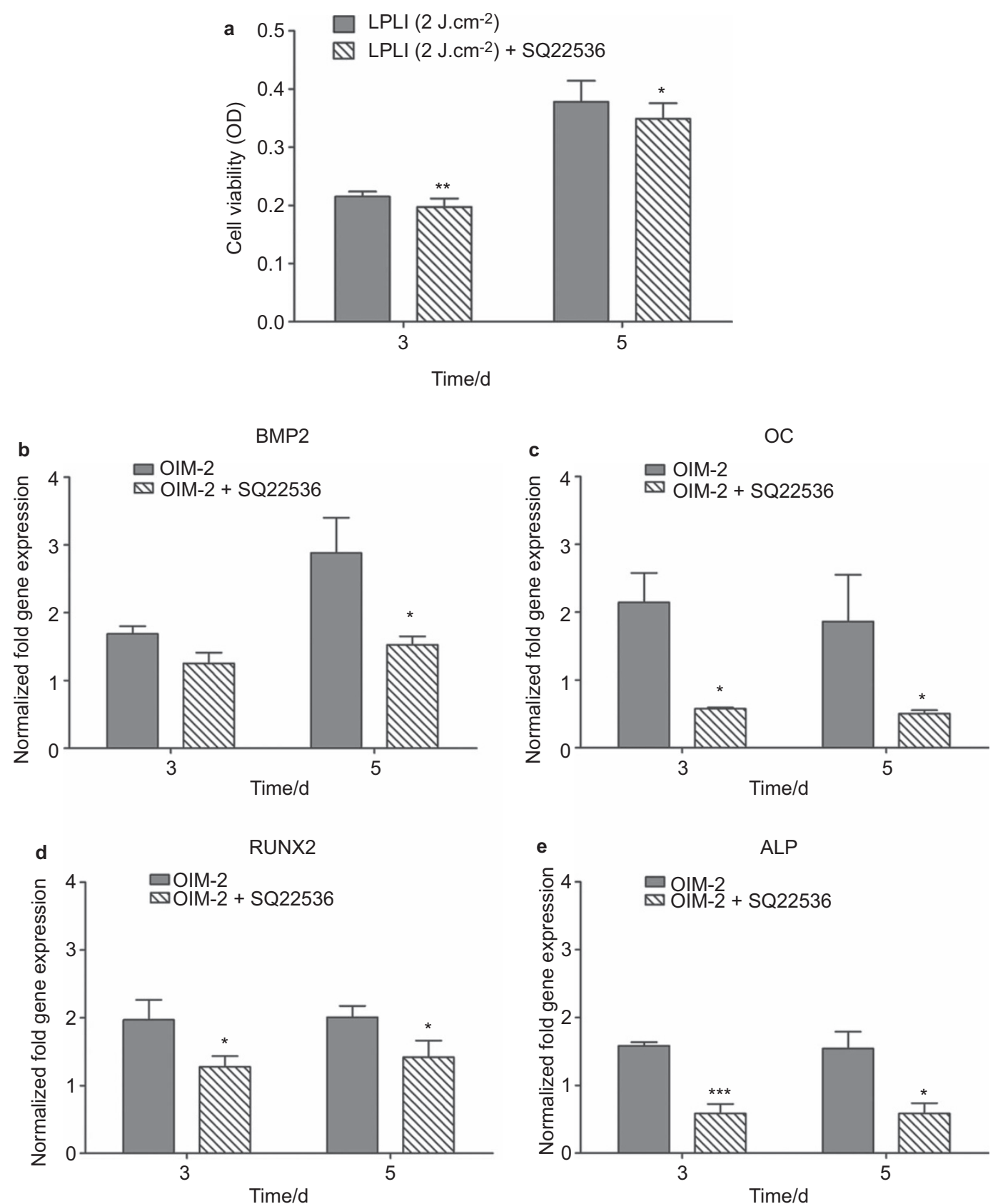

Figure 4 cAMP regulated the LPLI-mediated proliferation and osteogenic differentiation of hPDL cells. (a) hPDL cells were treated with $L P L I$ at $2 \mathrm{~J} \cdot \mathrm{cm}{ }^{-2}$ alone or in combination with SQ22536, and then an MTT assay was performed $(n=12)$. (b-e) The effect of CAMP signaling on the expression of osteogenic genes (BMP2, OC, RUNX2 and $A L P$ in sequence) by RT-PCR $(n=4)$. The data are shown as the mean \pm s.d., and the levels of statistical significance are indicated as follows: $* P<0.05, * * P<0.01$ and $* * * P<0.001$ relative to the LPLI $\left(2 \mathrm{~J} \cdot \mathrm{cm}^{-2}\right) / O \mathrm{IM}-2$ group. ALP, alkaline phosphatase; hPDL, human periodontal ligament; LPLI, low-power laser irradiation; MTT, 3-[4,5dimethylthiazol-2-yl]-2,5-diphenyltetrazolium bromide; OIM, osteo-induction medium; RT-PCR, reverse transcription-polymerase chain reaction; OD, optical density.

(Figure 3d). Overall, LPLI consistently increased BMP2, OC, RUNX2 and $A L P$ gene expression on day 3 at both energy doses $\left(2\right.$ and $\left.4 \mathrm{~J} \cdot \mathrm{cm}^{-2}\right)$, but the effect was not maintained when those genes were observed on day 5 with the exception of the BMP2 and $A L P$, which revealed a difference in cells treated with LPLI at $2 \mathrm{~J} \cdot \mathrm{cm}^{-2}$ at that time. Furthermore, no differences were observed between the two laser doses.

\section{cAMP modulates LPLI-induced cell proliferation and osteogenic} differentiation

cAMP is an important modulator of cell proliferation, differentiation, apoptosis and inflammation, and it also regulates LPLI signaling. To investigate whether cAMP is a critical factor involved in LPLI-induced cell proliferation and osteogenic gene expression, hPDL cells were treated with the adenylyl cyclase inhibitor SQ22536, and LPLIinduced cell proliferation and osteogenic gene expression were monitored. After SQ22536 treatment, the MTT activity of hPDL cells was similar to that of the control cells. Moreover, when compared with hPDL cells treated with LPLI at $2 \mathrm{~J} \cdot \mathrm{cm}^{-2}$, the MTT activity was significantly decreased on days 3 and 5 (Figure $4 \mathrm{a}$ ). Regarding the LPLIinduced effects on osteogenic genes, the LPLI-induced increase in the expression of osteogenic genes (BMP2, OC, RUNX2 and ALP) was significantly blocked following treatment with SQ22536 on days 
3 and 5 with the exception of BMP2 on day 3 (Figure $4 \mathrm{~b}-4 \mathrm{e}$ ). Remarkably, in the absence of LPLI, SQ22536 treatment did not affect hPDL cell proliferation and osteogenic gene expression (data not shown). Overall, these results indicate that LPLI-mediated hPDL cell proliferation and osteogenic differentiation may occur via the CAMP signaling pathway.

\section{DISCUSSION}

This study shows that the irradiation of hPDL cells with a low-power $660 \mathrm{~nm}$ GaAlAs red laser at various energy levels affects their physiological properties. hPDL cells have been considered the most important cell type in periodontal tissue for maintaining the periodontium. Others have suggested that hPDL cells possess the capacity for self-renewal and the ability to differentiate into cementoblasts and osteoblasts, which may lead to the repair of lost cementum and alveolar bone. ${ }^{18-20}$ For this reason, it is imperative to stimulate the proliferation and differentiation of PDL cells to achieve improved periodontal tissue regeneration.

Since the development and clinical application of low-power lasers in the 1970s, LPLI has been known to induce an effective biological response in living tissues. LPLI has been investigated for the characterization of PDL cells, including their attachment to root surfaces, ${ }^{21}$ relief from pain by inhibiting the production of prostaglandin E2. and interleukin- $1 \beta$ caused by tooth orthodontic treatment, ${ }^{22}$ and the reduction of plasminogen activator activity in response to mechanical stress. ${ }^{23}$ Although many studies have used growth factors ${ }^{24-25}$ and biomaterials ${ }^{26-27}$ in LPLI and PDL studies, only a few studies have examined the biological response of PDL cells to LPLI.

The effects of LPLI on hPDL cell proliferation remain controversial. In 2003, Kreisler et al. ${ }^{28}$ used a low-level diode laser ( $809 \mathrm{~nm}$ ) at energy fluences of $1.96,3.92$ and $7.84 \mathrm{~J} \cdot \mathrm{cm}^{-2}$ to irradiate hPDL cells. At each energy level, the irradiated hPDL cells had a higher rate of proliferation than the non-irradiated cells for up to $72 \mathrm{~h}$. In 2010, Choi et al. ${ }^{29}$ studied the effects of a low-power GaAlAs semiconductor laser $(810 \mathrm{~nm})$ on hPDL cells at energy fluences of 1.97, 3.94 and $5.91 \mathrm{~J} \cdot \mathrm{cm}^{-2}$. In this study, results from the MTT assay revealed that all three energy doses induced a gradual but insignificant increase in the rate of proliferation for up to $72 \mathrm{~h}$. In contrast, in this study, we used a low-power GaAlAs semiconductor laser with a wavelength within the visible spectrum $(660 \mathrm{~nm})$. We found that LPLI at 1 and $2 \mathrm{~J} \cdot \mathrm{cm}^{-2}$ induced a significant increase in proliferation at days 3 and 5 . Furthermore, irradiation energy greater than $2 \mathrm{~J} \cdot \mathrm{cm}^{-2}$ did not enhance hPDL cell proliferation. These findings are similar to those of Walsh ${ }^{30}$ who found that the stimulating effects of LPLI occurred at energy densities up to $4 \mathrm{~J} \cdot \mathrm{cm}^{-2}$, whereas the LPLI inhibitory effects occurred at higher energy doses. In summary, the increased rate of hPDL cell proliferation induced by LPLI may have potential applications in the promotion of cellular activity in localized periodontal sites.

ALP activity is a known biomarker used to evaluate initial osteogenic differentiation. Choi et al. ${ }^{29}$ published the only known study evaluating the effects of LPLI on ALP activity in hPDL cells. This study indicated that LPLI at $1.97,3.94$ and $5.91 \mathrm{~J} \cdot \mathrm{cm}^{-2}$ resulted in significantly increased ALP activity levels compared with non-irradiated control cells. However, a laser energy dose of only $3.94 \mathrm{~J} \cdot \mathrm{cm}^{-2}$ resulted in significantly different ALP activities at 48 and $72 \mathrm{~h}$. Interestingly, hPDL cells were not cultured in osteogenic induction medium, which mimics the bone repair environment. ${ }^{29}$ In all of the osteogenesis experiments in our study, we cultured hPDL cells in osteogenic induction medium, which provides a more suitable condition for evaluating the osteogenic differentiation of hPDL cells. The results showed that LPLI at $2 \mathrm{~J} \cdot \mathrm{cm}^{-2}$ significantly increased the ALP activity at days 3 and 5. These results suggest that LPLI potentially acts to enhance the osteogenic differentiation of hPDL cells.

We provide the first report of the osteogenic effects of LPLI on hPDL cells using gene expression and calcium deposition. Our results facilitate the determination of the precise effects of LPLI on the osteogenic differentiation process. These data combined with osteogenic functional assays and the expression of osteogenic genes indicate that hPDL cells have higher mineralization potential when cultured in OIM than control cells cultured in basal growth medium. Moreover, LPLI at 2 and $4 \mathrm{~J} \cdot \mathrm{cm}^{-2}$ consistently led to calcium deposition in hPDL cells. In addition, LPLI at 2 and $4 \mathrm{~J} \cdot \mathrm{cm}^{-2}$ consistently increased BMP2, OC, RUNX2 and ALP gene expression on day 3 , but this increase was only observed for BMP2 and $A L P$ at a dose of $2 \mathrm{~J} \cdot \mathrm{cm}^{-2}$ on day 5 . Interestingly, despite the ALP activity, Alizarin Red S staining, and real-time RT-PCR data, LPLI produced consistent effects with respect to the acceleration of the osteogenic differentiation of hPDL cells. However, the optimal treatment dose is unclear. Cells treated with LPLI at either 2 or $4 \mathrm{~J} \cdot \mathrm{cm}^{-2}$ exhibited increased performance; however, these increases were observed in different tests. We attribute this phenomenon to the complexity of osteogenic differentiation, a process that is regulated by many signaling pathways and mediators. Further investigation is required to determine the optimal treatment conditions for LPLI-mediated osteogenic differentiation.

cAMP is a crucial second messenger for many cellular processes, including cell proliferation, differentiation, apoptosis, and inflammation. ${ }^{31}$ Several studies, including our previous study, ${ }^{16}$ have reported that LPLI increases intracellular cAMP levels. ${ }^{32-33}$ In this study, the biological effects of LPLI were reversed after treatment with SQ22536, an adenylyl cyclase inhibitor. This finding indicates that LPLI may elevate the cAMP level to promote hPDL cell proliferation and osteogenic gene expression. For osteogenic differentiation, our data consistently showed that cAMP increased osteogenic gene expression, as previously reported. ${ }^{34-35}$ However, the role of cAMP in cell proliferation is controversial for different cell types. Some studies have reported that cAMP stimulates proliferation by modulating the $\mathrm{CAMP} /$ protein kinase A or mitogen-activated protein kinase pathways to regulate the transcription factor cAMP response elementbinding protein. ${ }^{36-37}$ Other studies have reported that cAMP negatively inhibits the cell proliferation through regulation of CDK4 or inhibition of cAMP response element-binding protein transcriptional function. ${ }^{38-39}$ Our previous study showed that the biological effects of LPLI may modulate intracellular cAMP levels and affect nuclear factorkappa B (NF- $\kappa \mathrm{B})$ activity. However, whether the LPLI-mediated biological effects on hPDL cells also work through the cAMP/NF- $\kappa B$ pathway is unclear. Further studies are necessary to clarify this matter.

\section{CONCLUSION}

In conclusion, a low-power GaAlAs 660-nm red laser enhanced the proliferation and osteogenic differentiation of hPDL cells via cAMP regulation. We also found that LPLI at $2 \mathrm{~J} \cdot \mathrm{cm}^{-2}$ improved hPDL cell proliferation. LPLI treatment was functionally and genetically confirmed to have potential osteogenic capability at 2 and $4 \mathrm{~J} \cdot \mathrm{cm}^{-2}$. Our results suggest that the non-invasive LPLI method may potentially be used to enhance periodontal tissue regeneration. Furthermore, understanding the molecular mechanisms of LPLI treatment in hPDL cells is necessary for future studies of the clinical application of LPLI to periodontal tissue regeneration. 


\section{ACKNOWLEDGEMENTS}

This study was supported by grants from the Kaohsiung Medical University of Taiwan (KMU-Q099018 and KMU-Q098025).

1 Nanci A. Ten Cate's oral histology: development, structure, and function. St Louis: Mosby, 2007.

2 Rygh P. Ultrastructural changes in tension zones of rat molar periodontium incident to orthodontic tooth movement. Am J Orthod 1976; 70(3): 269-281.

3 Christgau M, Caffesse RG, Schmalz G et al. Extracellular matrix expression and periodontal wound-healing dynamics following guided tissue regeneration therapy in canine furcation defects. J Clin Periodontol 2007; 34(8): 691-708.

4 Niemiec BA. Periodontal therapy. Top Companion Anim Med 2008; 23(2): 81-90.

5 Gottlow J, Nyman S, Lindhe J et al. New attachment formation in the human periodontium by guided tissue regeneration. Case reports. J Clin Periodontol 1986 13(6): 604-616.

6 Bowers GM, Chadroff B, Carnevale R et al. Histologic evaluation of new attachment apparatus formation in humans. Part I. J Periodontol 1989; 60(12): 664-674.

7 Bertolini GR, Artifon EL, Silva TS et al. Low-level laser therapy, at $830 \mathrm{~nm}$, for pain reduction in experimental model of rats with sciatica. Arq Neuropsiquiatr 2011; 69(2B): 356-359

8 Doshi-Mehta G, Bhad-Patil WA. Efficacy of low-intensity laser therapy in reducing treatment time and orthodontic pain: a clinical investigation. Am J Orthod Dentofacial Orthop 2012; 141(3): 289-297.

9 de Lima FM, Villaverde AB, Albertini R et al. Dual effect of low-level laser therapy (LLLT) on the acute lung inflammation induced by intestinal ischemia and reperfusion: action on anti- and pro-inflammatory cytokines. Lasers Surg Med 2011; 43(5): 410-420.

10 Pires D, Xavier M, Araujo T et al. Low-level laser therapy (LLLT; $780 \mathrm{~nm}$ ) acts differently on mRNA expression of anti- and pro-inflammatory mediators in an experimental model of collagenase-induced tendinitis in rat. Lasers Med Sci 2011; 26(1): 85-94.

11 Goncalves RV, Novaes RD, do Carmo Cupertino M et al. Time-dependent effects of lowlevel laser therapy on the morphology and oxidative response in the skin wound healing in rats. Lasers Med Sci 2012; 28(2): 383-390.

12 Melo VA, Anjos DC, Albuquerque Junior R et al. Effect of low level laser on sutured wound healing in rats. Acta Cir Bras 2011; 26(2): 129-134.

13 Hou JF, Zhang H, Yuan X et al. In vitro effects of low-level laser irradiation for bone marrow mesenchymal stem cells: proliferation, growth factors secretion and myogenic differentiation. Lasers Surg Med 2008; 40(10): 726-733.

14 Soleimani M, Abbasnia E, Fathi M et al. The effects of low-level laser irradiation on differentiation and proliferation of human bone marrow mesenchymal stem cells into neurons and osteoblasts_-an in vitro study. Lasers Med Sci 2012; 27(2): 423-430.

15 da Silva AP, Petri AD, Crippa GE et al. Effect of low-level laser therapy after rapid maxillary expansion on proliferation and differentiation of osteoblastic cells. Lasers Med Sci 2012; 27(4): 777-783.

16 Wu JY, Chen $\mathrm{CH}$, Wang $\mathrm{CZ}$ et al. Low-power laser irradiation suppresses inflammatory response of human adipose-derived stem cells by modulating intracellular cyclic AMP level and NF-kappaB activity. PloS ONE 2013; 8(1): e54067.

17 Wang $\mathrm{YH}, \mathrm{Ho} \mathrm{ML}$, Chang JK et al. Microporation is a valuable transfection method for gene expression in human adipose tissue-derived stem cells. Mol Ther 2009; 17(2): 302-308.

18 Silverio KG, Rodrigues TL, Coletta RD et al. Mesenchymal stem cell properties of periodontal ligament cells from deciduous and permanent teeth. J Periodontol 2010; 81(8): 1207-1215.

19 Seo BM, Miura M, Gronthos S et al. Investigation of multipotent postnatal stem cells from human periodontal ligament. Lancet 2004; 364(9429): 149-155.

20 Isaka J, Ohazama A, Kobayashi M et al. Participation of periodontal ligament cells with regeneration of alveolar bone. J Periodontol 2001; 72(3): 314-323.
21 Hakki SS, Korkusuz P, Berk G et al. Comparison of Er,Cr:YSGG laser and hand instrumentation on the attachment of periodontal ligament fibroblasts to periodontally diseased root surfaces: an in vitro study. J Periodontol 2010; 81(8): 1216-1225.

22 Shimizu N, Yamaguchi M, Goseki T et al. Inhibition of prostaglandin E2 and interleukin 1-beta production by low-power laser irradiation in stretched human periodontal ligament cells. J Dent Res 1995; 74(7): 1382-1388.

23 Ozawa Y, Shimizu N, Abiko Y. Low-energy diode laser irradiation reduced plasminogen activator activity in human periodontal ligament cells. Lasers Surg Med 1997; 21(5): 456-463.

$24 \mathrm{Yu}$ Y, Mu J, Fan Z et al. Insulin-like growth factor 1 enhances the proliferation and osteogenic differentiation of human periodontal ligament stem cells via ERK and JNK MAPK pathways. Histochem Cell Biol 2012; 137(4): 513-525.

25 Lee JH, Um S, Jang JH et al. Effects of VEGF and FGF-2 on proliferation and differentiation of human periodontal ligament stem cells. Cell Tissue Res 2012; 348(3): 475-484.

26 Zhou Y, Wu C, Xiao Y. The stimulation of proliferation and differentiation of periodontal ligament cells by the ionic products from $\mathrm{Ca}_{7} \mathrm{Si}_{2} \mathrm{P}_{2} \mathrm{O}_{16}$ bioceramics. Acta Biomater 2012; 8(6): 2307-2316.

27 Xia L, Zhang Z, Chen L et al. Proliferation and osteogenic differentiation of human periodontal ligament cells on akermanite and beta-TCP bioceramics. Eur Cell Mater 2011; 22: 68-82; discussion 83.

28 Kreisler M, Christoffers AB, Willershausen B et al. Effect of low-level GaAlAs laser irradiation on the proliferation rate of human periodontal ligament fibroblasts: an in vitro study. J Clin Periodontol 2003; 30(4): 353-358.

29 Choi EJ, Yim JY, Koo KT et al. Biological effects of a semiconductor diode laser on human periodontal ligament fibroblasts. J Periodontal Implant Sci 2010; 40(3): 105-110.

30 Walsh LJ. The current status of low level laser therapy in dentistry. Part 1. Soft tissue applications. Aust Dent J 1997; 42(4): 247-254.

31 Gether U. Uncovering molecular mechanisms involved in activation of $\mathrm{G}$ proteincoupled receptors. Endocrine reviews 2000; 21(1): 90-113.

32 Zungu IL, Hawkins Evans D, Abrahamse H. Mitochondrial responses of normal and injured human skin fibroblasts following low level laser irradiation-an in vitro study. Photochem Photobiol 2009; 85(4): 987-996.

33 Karu TI, Lobko VV, Lukpanova GG et al. [Effect of irradiation with monochromatic visible light on the cAMP content in mammalian cells.] Dokl Akad Nauk SSSR 1985; 281(5): 1242-1244. Russian.

34 Doorn J, Leusink M, Groen N et al. Diverse Effects of cyclic AMP variants on osteogenic and adipogenic differentiation of human mesenchymal stromal cells. Tissue Eng Part A 2012; 18(13/14): 1431-1442.

35 Siddappa R, Doorn J, Liu J et al. Timing, rather than the concentration of cyclic AMP, correlates to osteogenic differentiation of human mesenchymal stem cells. J Tissue Eng Regen Med 2010; 4(5): 356-365.

36 James MA, Lu Y, Liu Y et al. RGS17, an overexpressed gene in human lung and prostate cancer, induces tumor cell proliferation through the cyclic AMP-PKACREB pathway. Cancer Res 2009; 69(5): 2108-2116.

37 Takahashi H, Honma M, Miyauchi $\mathrm{Y}$ et al. Cyclic AMP differentially regulates cell proliferation of normal human keratinocytes through ERK activation depending on the expression pattern of B-Raf. Arch Dermatol Res 2004; 296(2): 74-82.

38 Rocha AS, Paternot S, Coulonval $\mathrm{K}$ et al. Cyclic AMP inhibits the proliferation of thyroid carcinoma cell lines through regulation of CDK4 phosphorylation. Mol Biol Cell 2008; 19(11): 4814-4825.

39 Inada A, Hamamoto Y, Tsuura Y et al. Overexpression of inducible cyclic AMP early repressor inhibits transactivation of genes and cell proliferation in pancreatic beta cells. Mol Cell Biol 2004; 24(7): 2831-2841.

(c) This work is licensed under a Creative Commons Attribution-NonCommercial-Share Alike 3.0 Unported License. To view a copy of this license, visit http://creativecommons. org/licenses/by-nc-sa/3.0 